\title{
EXTENSION OF EIGENFUNCTION-EXPANSION SOLUTIONS OF A FOKKER-PLANCK EQUATION-I. FIRST ORDER SYSTEM
}

\author{
JAMES P. JOHNSON and RichaRd A. SCOTT \\ Department of Applied Mechanics and Engineering Science, The University of Michigan, \\ Ann Arbor, Michigan, U.S.A.
}

(Received 19 July 1978)

\begin{abstract}
The work is concerned with eigenfunction-expansion solutions to the forward FokkerPlanck equation associated with a specific, non-linear, first-order system subject to white noise excitation. Using a digital computer, a substantial number of new terms in the expansions have been generated. With this new information, inverted Domb-Sykes plots revealed a pattern in the coefficients for certain ranges of values of the parameters. Through this pattern, Dingle's theory of terminants was used to recast the series into a more favorable computational form.
\end{abstract}

\section{INTRODUCTION}

Random excitation of non-linear systems is a challenging field of perennial interest (see Caughey's review [1]) and several approaches to obtaining solutions to associated FokkerPlanck equations have been developed. The present work is concerned with eigenfunctionexpansion procedures, as described by, for example, Atkinson [2]. Specifically, the work of Payne $[3,4]$ on a first-order, weakly non-linear system is considerably extended. This is achieved by means of tools developed elsewhere for the analysis and improvement of perturbation series (see Van Dyke [5]). Also, the theory of terminants developed by Dingle [6] is harnessed in the work.

The spirit of the approach is to obtain a sufficient number of computer generated terms that a pattern, if it exists, emerges. This pattern is then used to recast the expansion into a more favorable computational form. Using this approach, a substantial body of new information on the steady-state, mean square response of a specific first-order system to a white noise excitation is presented.

\section{EIGENFUNCTION-EXPANSION SOLUTIONS}

Following Payne $[3,4]$, the system considered is

$$
\frac{\mathrm{d} x}{\mathrm{~d} t}+x+\varepsilon x^{3}=n(t)
$$

where $\varepsilon$ is a small parameter and $n(t)$ is a white noise process with the properties: (i) $n\left(t_{i}\right)$, $i=1,2 \ldots$, are mutually independent and (ii) $n(t)$ has a Gaussian probability distribution with $E[n(t)]=0, E[n(t) n(s)]=2 D \delta(t-s), E$ denoting expected value, $\delta$ being the delta function, and $D$ a constant which measures the white noise intensity. The response $x$ is modeled as a Markov process and the forward Fokker-Planck equation associated with equation (1) is

$$
\frac{\partial p}{\partial t}=\frac{\partial}{\partial x}\left[\left(x+\varepsilon x^{3}\right) p\right]+D \frac{\partial^{2} p}{\partial x^{2}} \quad t>0
$$

where $p=p\left(x, t \mid x_{0} ; \varepsilon\right)$ is the transition probability density and satisfies

$$
\int_{-\infty}^{\infty} p\left(x, t \mid x_{0} ; \varepsilon\right) \mathrm{d} x=1
$$




$$
\operatorname{Lim}_{t \rightarrow 0} \int_{-\infty}^{\infty} p\left(x, t \mid x_{0} ; \varepsilon\right) h(x) \mathrm{d} x=h\left(x_{0}\right)
$$

where $h(x)$ is an arbitrary continuous function. Also, on integrating (2) with respect to $x$ and using (3), it follows that

$$
\operatorname{Lim}_{x \rightarrow \pm \infty}\left[D \frac{\partial p}{\partial x}+\left(x+\varepsilon x^{3}\right) p\right]=0
$$

Introducing the variable $\zeta=x / \sqrt{2 D}$, the expression

$$
p\left(\zeta, t \mid \zeta_{0} ; \varepsilon\right)=p_{s}(\zeta ; \varepsilon) \sum_{n=0}^{\infty} v_{n}\left(\zeta_{0} ; \varepsilon\right) v_{n}(\zeta ; \varepsilon) \exp \left[-\lambda_{n}(\varepsilon)\right] t
$$

where the subscript $s$ stands for 'steady-state', is a solution to (2) provided

$$
\left[\frac{\mathrm{d}^{2}}{\mathrm{~d} \zeta^{2}}-2 \zeta \frac{\mathrm{d}}{\mathrm{d} \zeta}+2 \lambda_{n}(\varepsilon)\right] v_{n}(\zeta ; \varepsilon)=4 \varepsilon D \zeta^{3} \frac{\mathrm{d}}{\mathrm{d} \zeta} v_{n}(\zeta ; \varepsilon)
$$

It can readily be shown that the steady state solution is

$$
p_{s}(\zeta ; \varepsilon)=\left[\exp \left(-\zeta^{2}-\varepsilon D \zeta^{4}\right)\right]\left[\int_{-\infty}^{\infty} \exp \left(-\eta^{2}-\varepsilon D \eta^{4}\right) \mathrm{d} \eta\right] .
$$

Also, by definition

$$
v_{0}\left(\zeta_{0} ; \varepsilon\right)=1, v_{0}(\zeta ; \varepsilon)=1, \lambda_{0}(\varepsilon)=0 .
$$

Payne has shown that (2) and (7) constitute an eigenvalue problem. Moreover, he gave conditions on $p$, assumed to be true here, under which the spectrum would be discrete. Thus, (6) constitutes an eigenfunction-expansion solution. The eigenfunctions have the orthogonality property

$$
\int_{-\infty}^{\infty} p_{s}(\zeta ; \varepsilon) v_{n}(\zeta ; \varepsilon) v_{m}(\zeta ; \varepsilon) \mathrm{d} \zeta=\delta_{n m}
$$

where $\delta_{n m}$ denotes the Kronecker delta.

When $\varepsilon=0,(1)$ is linear and then (7) reduces to Hermite's equation, the solution to which is

$$
\begin{aligned}
v_{n 0}(\zeta) & =H_{n}(\zeta) / \sqrt{2^{n} n !} \\
\lambda_{n 0} & =n
\end{aligned}
$$

where the Hermite polynomial is defined by

$$
H_{n}(\zeta)=n ! \sum_{k=0}^{k<n / 2} \frac{(-1)^{k}}{k !(n-2 k) !}(2 \zeta)^{n-2 k}
$$

In (11) and (12) the additional subscript 0 designates the $\varepsilon=0$ case. Equations (8) and (10) now combine to give

$$
\int_{-\infty}^{\infty} p_{s}(\zeta) v_{n 0}(\zeta) v_{m 0}(\zeta) \mathrm{d} \zeta=\delta_{n m}
$$

where

$$
\sqrt{\pi} p_{s}(\zeta)=\mathrm{e}^{-\zeta^{2}}
$$

When $\varepsilon \neq 0$, but small, one may think of the process as perturbing the eigenfunctions $v_{n 0}$ and eigenvalues $\lambda_{n 0}$. Within this conceptual framework the following expansions are set forth (see Courant and Hilbert [7]):

$$
\begin{gathered}
v_{n}(\zeta ; \varepsilon)=\sum_{j=0}^{\infty} v_{n}(\zeta) \varepsilon^{j} \\
\lambda_{n}(\varepsilon)=\sum_{j=0}^{\infty} \lambda_{n j} \varepsilon^{j} .
\end{gathered}
$$


Substituting Eqs. (16) and (17) into Eq. (7), and setting the coefficients of the various powers of $\varepsilon$ separately to zero yields

$$
L v_{n j}+2 \sum_{i, k=0}^{\infty} \delta(j-i-k) \lambda_{n i} v_{n k}=4 D \zeta^{3} v_{n(j-1)}^{\prime} \quad j=0,1,2, \ldots
$$

where the operator $L$ is given by

$$
L=\frac{\mathrm{d}^{2}}{\mathrm{~d} \zeta^{2}}-2 \zeta \frac{\mathrm{d}}{\mathrm{d} \zeta}
$$

and the prime denotes differentiation w.r.t. $\zeta$. Here, and throughout, any quantity with a negative subscript is to be understood as having a value of zero.

Still another expansion is introduced at this stage, namely

$$
v_{n i}(\zeta)=\sum_{j=0}^{\infty} a_{n i j} v_{j 0}(\zeta) \quad i=0,1,2, \ldots
$$

where the $a_{n i j}$ are constants to be determined and the $v_{j 0}$ are the scaled Hermite polynomials given by equation (11). Using their orthogonality property, (14), the $a_{n i j}$ may be written

$$
a_{n i j}=\left(v_{n i}, v_{j 0}\right)
$$

where an inner product notation has been introduced, namely

$$
(u, v)=\int_{-\infty}^{\infty} p_{s}(\zeta) u(\zeta) v(\zeta) \mathrm{d} \zeta .
$$

Recursion relations will now be developed. Multiplying (18) by $p_{s}(\zeta) v_{l 0}(\zeta)$ and integrating gives

$$
\left(v_{l 0}, L v_{n j}\right)+2 \sum_{i, k=0}^{\infty} \delta(j-i-k) \lambda_{n i}\left(v_{l 0}, v_{m k}\right)=4 D\left(v_{l 0}, \zeta^{3} v_{n(j-1)}^{\prime}\right) \quad j=0,1,2, \ldots
$$

$L$ is a self adjoint operator, i.e. $(L u, v)=(u, L v)$. Using this together with equation (7) with $\varepsilon=0$ and (21), (23) may be written

$$
2 \sum_{i, k=0}^{\infty} \delta(j-i-k) \lambda_{n i} a_{n k l}-2 \lambda_{10} a_{n j l}=4 D\left(v_{l 0}, \zeta^{3} v_{n(j-1)}^{\prime}\right) \quad j=0,1,2, \ldots
$$

To obtain recursion relations for the $\lambda$ 's, consider $l=n$. Using a property of the delta function, (24) may be written

$$
\sum_{i=1}^{j} \lambda_{n i} a_{n(j-i) n}=2 D\left(v_{n 0}, \zeta^{3} v_{n(j-i)}^{\prime}\right) \quad j=0,1,2, \ldots
$$

Equations (12) and (25) give the recursion relations

$$
\begin{aligned}
& \lambda_{n 0}=n \\
& \lambda_{n 1}=2 D\left(v_{n 0}, \zeta^{3} v_{n 1}^{\prime}\right) \\
& \lambda_{n j}=2 D\left(v_{n 0}, \zeta^{3} v_{n j}^{\prime}\right)-\sum_{i=1}^{j-1} \lambda_{n i} a_{n(j-i) n} \quad j \geqslant 2 .
\end{aligned}
$$

Consider now $l \neq n$. Equations (12) and (24) give

$$
(n-l) a_{n j l}+\sum_{i=1}^{\infty} \lambda_{n i} a_{n(j-i) l}=2 D\left(v_{l 0}, \zeta^{3} v_{n(j-1)}^{\prime}\right) \quad j=0,1,2, \ldots
$$

from which it follows that

$$
\begin{gathered}
a_{n 0 l}=\delta_{n l} \\
a_{n j l}=\frac{1}{n-l}\left[\begin{array}{c}
\left.2 D\left(v_{l 0}, \zeta^{3} v_{m(j-1)}^{\prime}\right)-\sum_{i=1}^{j} \lambda_{n i} a_{n(j-i) l}\right] \\
n \neq l, j=1,2, \ldots
\end{array}\right.
\end{gathered}
$$


The recursion relation for $a_{\text {nin }}$ must now be calculated. Expanding exp $\left(-\varepsilon D \zeta^{4}\right)$ and $\exp \left(-\varepsilon D \eta^{4}\right)$ in (8) in Maclaurin series can be shown to lead to

$$
\begin{aligned}
p_{s}(\zeta ; \varepsilon) & =\frac{\mathrm{e}^{-\zeta^{2}}}{\sqrt{\pi}}\left[\sum_{n=0}^{\infty} \frac{\left(-D \zeta^{4}\right)^{n}}{n !} \varepsilon^{n}\right] /\left[\sum_{n=0}^{\infty} \frac{(-D \varepsilon)^{n}}{n !} \int_{-\infty}^{\infty} \eta^{4 n} \mathrm{e}^{-\zeta^{2}} \mathrm{~d} \eta\right] \\
& =\frac{\mathrm{e}^{-\zeta^{2}}}{\sqrt{\pi}}\left[\sum_{n=0}^{\infty} \frac{\left(-D \zeta^{4}\right)^{n}}{n !} \varepsilon^{n}\right] /\left[\sum_{n=0}^{\infty} \frac{(-D)^{n}[1.3 .5 \cdot \cdots(4 n-1)]}{2^{2 n} n !} \varepsilon^{n}\right] .
\end{aligned}
$$

When the indicated division of series is performed, (31) can be written

$$
p_{s}(\zeta ; \varepsilon)=\frac{\mathrm{e}^{-\zeta^{2}}}{\sqrt{\pi}} \sum_{n, m=0}^{\infty} \frac{\left(-D \zeta^{4}\right)^{n}}{n !} b_{m} \varepsilon^{n+m}
$$

where

$$
b_{0}=1, b_{m}=-\sum_{j=1}^{m} \frac{(-D)^{j}[1.3 .5 \cdot \cdots(4 j-1)]}{2^{2 j} j !} b_{m-j} \quad m \geqslant 1 .
$$

Equations (10), (16), (20) and (32) give, on interchanging summation and integration

$$
\sum_{i, j, k, l=0}^{\infty} \frac{(-D)^{i+j} b_{j}}{i !} \sum_{r, s=0}^{\infty} a_{n k r} a_{m l s}\left(\zeta^{4 i} v_{r 0}, v_{s 0}\right) \varepsilon^{i+j+k+1}=\delta_{n m} .
$$

Let $m=n$ and group according to powers of $\varepsilon$, denoting the coefficient of $\varepsilon^{N}$ by $c_{N}$. To satisfy (34) with $n=m$, it must follow that $c_{0}=1$, which can readily be shown to be true, and $c_{N}=0$, $N>0$. This latter condition together with (34), requires that

$$
\sum_{i, j, k, l=0}^{N} \delta(N-i-j-k-l) \frac{(-D)^{i+j}}{i !} b_{j} \sum_{r, s=0}^{\infty} a_{n k r} a_{n l s}\left(l_{s}^{4 i} v_{r 0}, v_{s 0}\right)=0 \quad N>0 .
$$

By systematically isolating terms of the form $a_{n k n}$ and $a_{n l n}$ in (35), the recursion relation for $a_{n N n}$ can be obtained as

$$
\begin{aligned}
a_{n N n}= & -\frac{1}{2} \sum_{i, j, k, l=0}^{N} \delta(N-i-j-k-l) \frac{(-D)^{i+j}}{i !} b_{j}\left[\sum_{r, s=0}^{n-1} a_{n k r} a_{n l s}\left(\zeta^{4 i} v_{r 0}, v_{s 0}\right)\right. \\
& \left.+\sum_{r=0}^{n-1} \sum_{s=n+1}^{\infty}\left(a_{n k r} a_{n l s}+a_{n k s} a_{n l}\right)\left(\zeta^{4 i} v_{r 0}, v_{s 0}\right)+\sum_{r, s=n+1}^{\infty} a_{n k r} a_{n l s}\left(\zeta^{4 i} v_{r 0}, v_{s 0}\right)\right] \\
& -\frac{1}{2} \sum_{i, j=0}^{N} \sum_{k, l=0}^{N-1} \delta(N-i-j-k-l) \frac{(-D)^{i+j}}{i !} b_{j}\left[\sum_{r=0}^{n-1}\left(a_{n k r} a_{n l n}+a_{n k n} a_{n t r}\right)\left(\zeta^{4 i} v_{r 0}, v_{n 0}\right)\right. \\
& \left.+a_{n k n} a_{n l n}\left(\zeta^{4 i} v_{n 0}, v_{n 0}\right)+\sum_{r=n+1}^{\infty}\left(a_{n k n} a_{n l r}+a_{n k r} a_{n l n}\right)\left(\zeta^{4 i} v_{r 0}, v_{n 0}\right)\right] \quad N>0 .
\end{aligned}
$$

Finally, the requirements of (9), together with (16), (17) and (20), can be shown to yield

$$
\begin{array}{ll}
\lambda_{0 j}=0 & \text { all } j \\
a_{0 i j}=0 & i>0 .
\end{array}
$$

The recursion relations for the eigenvalues and the eigenfunctions can be employed once the various inner product expressions have been evaluated, a task postponed until later. First an expression for the mean square response of the system will be developed. The autocorrelation function of the response, which is assumed to be a stationary Markov process, is given by, where $\tau$ denotes a time lag

$$
R_{x x}(\tau)=2 D E[\zeta(t), \zeta(t+\tau)]=2 D \iint_{-\infty}^{\infty} \zeta_{t+\tau} \zeta_{t} p\left(\zeta_{t+\tau}, t+\tau \mid \zeta_{t}, t\right) p\left(\zeta_{t}, t \mid \zeta_{0}\right) \mathrm{d} \zeta_{t} \mathrm{~d} \zeta_{t+\tau}
$$

or, noting that a stationary process is independent of a shift in the time origin

$$
R_{x x}(\tau)=2 D \operatorname{Lim}_{t \rightarrow \infty} \iint_{-\infty}^{\infty} \zeta_{t+\tau} \zeta_{t} p\left(\zeta_{t+t}, t+\tau \mid \zeta_{t}, t\right) p\left(\zeta_{t}, t \mid \zeta_{0}\right) \mathrm{d} \zeta_{t} \mathrm{~d} \zeta_{t+\tau}
$$


Using (6) and taking the limit inside the integral, (39) gives on rearranging

$$
R_{x x}(\tau)=2 D \sum_{n=0}^{\infty} \alpha_{n}^{2}(\varepsilon) \exp \left[-\lambda_{n}(\varepsilon) \tau\right]
$$

where

$$
\alpha_{n}(\varepsilon)=\int_{-\infty}^{\infty} \zeta p_{s}(\zeta ; \varepsilon) v_{n}(\zeta ; \varepsilon) \mathrm{d} \zeta
$$

By means of (6), (16) and (20), Eq. (41) can be written

$$
\alpha_{n}(\varepsilon)=\sum_{i, j, k, l=0}^{\infty} a_{n k l} \frac{(-D)^{i} b_{j}}{i !} \varepsilon^{i+j+k} \int_{-\infty}^{\infty} p_{s}(\zeta) \zeta^{4 i+1} v_{l 0}(\zeta) \mathrm{d} \zeta
$$

Noting that $v_{10}(\zeta)=\sqrt{2} \zeta$ and using (22), (42) gives

$$
\alpha_{n}(\varepsilon)=\sum_{i, j, k, l=0}^{\infty} a_{n k l} \frac{(-D)^{i}}{\sqrt{2} i !} b_{j}\left(\zeta^{4 l} v_{10}, v_{t 0}\right) \varepsilon^{i+j+k}
$$

Setting $\tau=0$ and employing $(40)$ and (43), the mean square response is obtained as

$$
E\left[x^{2}\right]=\sum_{m=0}^{\infty} a_{m} \varepsilon^{m}
$$

where

$$
\begin{gathered}
a_{m}=2 D \sum_{n=0}^{\infty} \sigma_{n m} \\
\sigma_{n m}=\frac{1}{2}\left[\sum_{i, j, k, F, q, r=0}^{m} \delta(m-i-j-k-p-q-r) \frac{b_{j} b_{q}}{i ! p !}(-D)^{i+p}\right] \\
\times\left[\sum_{l, s=0}^{\infty} a_{n k l} a_{n, r s}\left(\zeta^{4 i} v_{10}, v_{10}\right)\left(\zeta^{4 p} v_{10}, v_{s 0}\right)\right]
\end{gathered}
$$

The recursion relations involve inner products of the form $\left(v_{i 0}, \zeta^{3} v_{j n}^{\prime}\right)$ and $\left(\zeta^{i} v_{j 0}, v_{k 0}\right)$ and these will now be evaluated. Noting that the Hermite polynomials satisfy

$$
H_{0}^{\prime}(\zeta)=0, H_{n}^{\prime}(\zeta)=2 n H_{n-1}(\zeta)
$$

it follows from (11) and (20) that in fact all the inner products can be reduced to an evaluation of the general form $\left(\zeta^{i} v_{j 0}, v_{k 0}\right)$, which can be written

$$
\left(\zeta^{i} v_{j 0}, v_{k 0}\right)=\frac{1}{\sqrt{2^{j+k} j ! k !}} \int_{-\infty}^{\infty} p_{s}(\zeta) \zeta_{\zeta}^{i} H_{j}(\zeta) H_{k}(\zeta) \mathrm{d} \zeta .
$$

Using (13) and (15), (47) can be shown to yield

$$
\left(\zeta^{i} v_{j 0}, v_{k 0}\right)=\sqrt{2^{j+k} j ! k !} \sum_{i=0}^{i<j / 2} \sum_{m=0}^{m<k / 2} \frac{E(i+j+k-2(l+m))}{(-4)^{i+m}(j-2) !(k-2 m) ! ! ! m !}
$$

where

$$
E(N)=\left\{\begin{array}{l}
0, N \text { odd } \\
1, N=0 \\
\frac{1.3 .5 \ldots(N-1)}{2^{N / 2}} \quad N \text { even. }
\end{array}\right.
$$

By means of (48), the recursion relations for the eigenvalues and eigenfunctions, and the perturbation expansion for the mean square response can be calculated to any desired order using a digital computer. 


\section{COMPUTER EXTENSION OF PERTURBATION SOLUTIONS}

The recursion relations to obtain the eigenvalues and the eigenfunction expansion coefficients and the formulas for the perturbation expansion of the mean square response were programmed on the Ford Motor Company Honeywell 6000 computer in the STRAN (Structured Fortran) computer language. Not surprisingly, the programming involved considerable effort and the authors would be pleased to supply interested readers with details on program listings and organization.

Payne [4] presented results for the present system out to the second order. Here the perturbations were carried out to the eighth ordert for values of the white noise intensity $D$ ranging between 0.0001 to 100 and this substantial body of new information is given in Table 1. Agreement with Payne to the second order should be noted $\ddagger$

Table 1. Mean square response perturbation coefficients for various values of the intensity $D$

\begin{tabular}{|c|c|c|c|}
\hline & $D=0.0001$ & $D=0.001$ & $D=0.01$ \\
\hline \multirow[t]{2}{*}{$\begin{array}{l}a_{0} \\
a_{1} \\
a_{2} \\
a_{3} \\
a_{4} \\
a_{5} \\
a_{6} \\
a_{7} \\
a_{8}\end{array}$} & $\begin{array}{r}0.1000000 \mathrm{E}-03 \\
-0.1500450 \mathrm{E}-03 \\
-0.4874324 \mathrm{E}-03 \\
-0.4106030 \mathrm{E}-02 \\
-0.5262706 \mathrm{E}-01 \\
-0.8891938 \mathrm{E}-00 \\
-0.1853723 \mathrm{E} 02 \\
-0.4588644 \mathrm{E} 03 \\
-0.1314790 \mathrm{E} 05\end{array}$ & $\begin{array}{r}0.1000000 \mathrm{E}-02 \\
-0.1504500 \mathrm{E}-02 \\
-0.4868214 \mathrm{E}-02 \\
-0.4104062 \mathrm{E}-01 \\
-0.5261046 \mathrm{E}-00 \\
-0.8889810 \mathrm{E}-01 \\
-0.1853363 \mathrm{E} 03 \\
-0.4587894 \mathrm{E} 04 \\
-0.1314604 \mathrm{E} 06\end{array}$ & $\begin{array}{c}0.1000000 \mathrm{E}-01 \\
-0.1545000 \mathrm{E}-01 \\
-0.14803938 \mathrm{E}-01 \\
-0.4084888 \mathrm{E} 00 \\
-0.5244580 \mathrm{E} 01 \\
-0.8868638 \mathrm{E} 02 \\
-0.1849780 \mathrm{E} 04 \\
-0.4580418 \mathrm{E} 05 \\
-0.1312752 \mathrm{E} 07\end{array}$ \\
\hline & $D=0.1$ & $D=0.2$ & $D=0.5$ \\
\hline \multirow[t]{2}{*}{$\begin{array}{l}a_{0} \\
a_{1} \\
a_{2} \\
a_{3} \\
a_{4} \\
a_{5} \\
a_{6} \\
a_{7} \\
a_{8}\end{array}$} & $\begin{array}{r}0.1000000 \mathrm{E} 00 \\
-0.1950000 \mathrm{E} 00 \\
-0.3843750 \mathrm{E} 00 \\
-0.3981656 \mathrm{E} 01 \\
-0.5082874 \mathrm{E} 02 \\
-0.8670164 \mathrm{E} 03 \\
-0.1815437 \mathrm{E} 05 \\
-0.4508292 \mathrm{E} 06 \\
-0.1294787 \mathrm{E} 08\end{array}$ & $\begin{array}{r}0.2000000 \mathrm{E} 00 \\
-0.4800000 \mathrm{E} 00 \\
-0.4200000 \mathrm{E} 00 \\
-0.8424000 \mathrm{E} 01 \\
-0.9621540 \mathrm{E} 02 \\
-0.1703229 \mathrm{E} 04 \\
-0.3555803 \mathrm{E} 05 \\
-0.8868724 \mathrm{E} 06 \\
-0.2551653 \mathrm{E} 08\end{array}$ & $\begin{array}{r}0.5000000 \mathrm{E} 00 \\
-0.1875000 \mathrm{E} 01 \\
-0.3703125 \mathrm{E} 01 \\
-0.4756640 \mathrm{E} 02 \\
-0.1294970 \mathrm{E} 00 \\
-0.6158097 \mathrm{E} 04 \\
-0.5913441 \mathrm{E} 05 \\
-0.2460330 \mathrm{E} 07 \\
-0.5595717 \mathrm{E} 08\end{array}$ \\
\hline & $D=0.7$ & $D=1$ & $D=5$ \\
\hline \multirow[t]{2}{*}{$\begin{array}{l}a_{0} \\
a_{1} \\
a_{2} \\
a_{3} \\
a_{4} \\
a_{5} \\
a_{6} \\
a_{7} \\
a_{8}\end{array}$} & $\begin{array}{r}0.7000000 \mathrm{E} 00 \\
-0.3255000 \mathrm{E} 01 \\
-0.1211438 \mathrm{E} 02 \\
-0.1355898 \mathrm{E} 03 \\
-0.8872697 \mathrm{E} \mathrm{03} \\
-0.2135682 \mathrm{E} 05 \\
-0.1454986 \mathrm{E} 06 \\
-0.7849864 \mathrm{E} 07 \\
-0.2337453 \mathrm{E} 08\end{array}$ & $\begin{array}{r}0.1000000 \mathrm{E} 01 \\
-0.6000000 \mathrm{E} 01 \\
0.3750000 \mathrm{E} 02 \\
-0.4860000 \mathrm{E} 03 \\
0.6505884 \mathrm{E} 04 \\
-0.1401909 \mathrm{E} 06 \\
0.2938146 \mathrm{E} 07 \\
-0.8865712 \mathrm{E} 08 \\
0.2493584 \mathrm{E} 10\end{array}$ & $\begin{array}{r}0.5000000 \mathrm{E} 01 \\
-0.1200000 \mathrm{E} 03 \\
0.4597501 \mathrm{E} 04 \\
-0.2647349 \mathrm{E} 06 \\
0.2037707 \mathrm{E} 08 \\
-0.1976074 \mathrm{E} 10 \\
0.2317035 \mathrm{E} 12 \\
-0.3188347 \mathrm{E} 14 \\
0.4928034 \mathrm{E} 16\end{array}$ \\
\hline & $D=10$ & $D=100$ & \\
\hline $\begin{array}{l}a_{0} \\
a_{1} \\
a_{2} \\
a_{3} \\
a_{4} \\
a_{5} \\
a_{6} \\
a_{7} \\
a_{8}\end{array}$ & $\begin{array}{r}0.1000000 \mathrm{E} 02 \\
-0.4650000 \mathrm{E} 03 \\
0.3625126 \mathrm{E} 05 \\
-0.4186026 \mathrm{E} 07 \\
0.6461296 \mathrm{E} 09 \\
-0.1255596 \mathrm{E} 12 \\
0.2947952 \mathrm{E} 14 \\
-0.8118050 \mathrm{E} 16 \\
0.2510452 \mathrm{E} 19\end{array}$ & $\begin{array}{r}0.1000000 \mathrm{E} 03 \\
-0.4515000 \mathrm{E} 05 \\
0.3569202 \mathrm{E} 08 \\
-0.4139694 \mathrm{E} 11 \\
0.6407094 \mathrm{E} 14 \\
-0.1247123 \mathrm{E} 18 \\
0.2931510 \mathrm{E} 21 \\
-0.8079800 \mathrm{E} 24 \\
0.2499916 \mathrm{E} 28\end{array}$ & \\
\hline
\end{tabular}

After this order, computer costs became prohibitively high.

$\ddagger$ The authors confirmed with Payne in a private communication that there is a typographical error in Reference [4]. The term $\lambda n_{1} a_{n n}$, in his notation, should be subtracted from 48 . 
These results, though valuable in themselves, can be made considerably more useful by means of the theory of terminants advanced by Dingle [6], and recently used by Buchanan [8] in a study on improvement of series representations. A basic aim of Dingle's work is the analysis and improvement of divergent asymptotic series, and inspection of Table 1 clearly shows that the series at hand are divergent. It should be noted that the sign pattern of the series changes from a single-sign series for small values of the intensity $D$ to an alternatingsign series for large values of $D$, with the exception of the range $0.2<D<1.0$. These values represent a transition region and, presumably, with more terms of the series available a stable pattern would emerge. They will not be pursued any further in this work. The overall sign pattern indicates that for small values of $D$, the expansion contains a Stoke's discontinuity, but that this does not occur for large values of $D$.

Further insight can be gained by constructing inverted Domb-Sykes plots, that is, plots of $a_{n-1} / a_{n}$ versus $1 / n$, a feature impossible before this work since sufficient data was not available. Figure 1 shows such a plot for $D=0.0001$, and is a typical result in that the

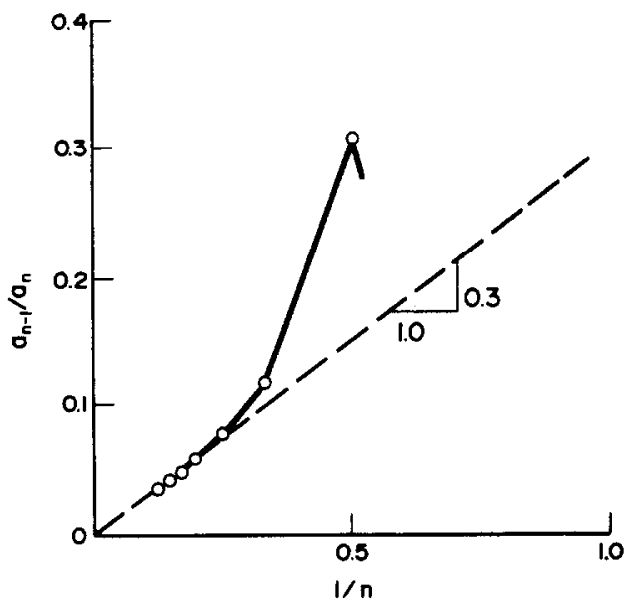

Fig. 1. Inverted Domb-Sykes plot for $D=0.0001$.

establishing of a linear relationship for large values of $n$ is becoming apparent. The best-fit slope for that relationship is indicated by the dashed line on the figure. This behavior is reproduced by taking $a_{n}=c(s)^{-n} n$ !, where $c$ is a constant and $s$ stands for slope, but following related work of Bender and $\mathrm{Wu}$ [9] on asymptotic series, more flexibility is obtained by taking $a_{n}=c(s)^{-n}(n+\alpha)$ !, where $0 \leqslant \alpha \leqslant 1$. Note that for large values of $n$ this gives a very good approximation to a straight line on an inverted Domb-Sykes plot. The parameters $c, s$ and $\alpha$ are to be determined using the higher order terms in the series. Adopting this procedure the general form of the series coefficients can be determined and is given in Table 2.

With this information the theory of terminants can now be employed. Consider the

Table 2. General form of the coefficients of the asymptotic series representation of the mean square response for various values of the intensity D

\begin{tabular}{lc}
\hline$D$ & $n$-th order coefficient $a_{n}$ \\
\hline 0.0001 & $-0.720118 \mathrm{E}-05 \times(1 / 0.3)^{n}\left(n+\frac{1}{2}\right) !$ \\
0.001 & $-0.719998 \mathrm{E}-04 \times(1 / 0.3)^{(}\left(n+\frac{1}{2}\right) !$ \\
0.01 & $-0.718805 \mathrm{E}-03 \times(1 / 0.3)^{n}\left(n+\frac{1}{2}\right) !$ \\
0.1 & $-0.707305 \mathrm{E}-02 \times(1 / 0.3)^{n}\left(n+\frac{1}{2}\right) !$ \\
5 & $0.491496 \mathrm{E} 01 \times(-1 / 0.05)^{n} n !$ \\
10 & $0.977591 \mathrm{E} 01 \times(-1 / 0.025)^{n} n !$ \\
100 & $0.972859 \mathrm{E} 02 \times(-1 / 0.0025)^{n} n !$ \\
\hline
\end{tabular}


case $D=0.0001$. The series can be written

$$
E\left[x^{2}\right]=\sum_{n=0}^{5} a_{n} \varepsilon^{n}-(0.720118 E-05) \sum_{n=6}^{\infty}\left(n+\frac{1}{2}\right) !\left(\frac{\varepsilon}{0.3}\right)^{n} .
$$

Using Dingle's terminant for a single-sign asymptotic series, equation (50) can be written in the much more useful form

$$
E\left[x^{2}\right]=\sum_{n=0}^{5} a_{n} \varepsilon^{n}-(0.720118 E-05)\left(6+\frac{1}{2}\right) !\left(\frac{\varepsilon}{0.3}\right)^{6} \pi_{6+1 / 2}\left(-\frac{0.3}{\varepsilon}\right)
$$

where the terminant $\pi$, a tabulated function, is given by

$$
\Pi_{m}(-x)=\frac{1}{m !} P \int_{0}^{\infty} \frac{\zeta^{m} \mathrm{e}^{-\zeta}}{1-\zeta / x} \mathrm{~d} \zeta
$$

$P$ denoting principal value. The results for the other values of $D$ are given in Table 3 .

Table 3. Terminated asymptotic series representing the mean square response for various values of the intensity $D$

\begin{tabular}{lc}
\hline$D$ & $E\left[x^{2}\right]$ \\
0.001 & $\sum_{n=0}^{5} a_{n} \varepsilon^{n}-(0.719998 \mathrm{E}-04)\left(6+\frac{1}{2}\right) !\left(\frac{\varepsilon}{0.3}\right)^{6} \pi_{6+1 / 2}(-0.3 / \varepsilon)$ \\
0.01 & $\sum_{n=0}^{5} a_{n} \varepsilon^{n}-\left(0.718805 \mathrm{E}-03\left(6+\frac{1}{2}\right) !\left(\frac{\varepsilon}{0.3}\right)^{6} \pi_{6+1 / 2}(-0.3 / \varepsilon)\right.$ \\
0.1 & $\sum_{n=0}^{5} a_{n} \varepsilon^{n}-(0.707305 \mathrm{E}-02)\left(6+\frac{1}{2}\right) !\left(\frac{\varepsilon}{0.3}\right)^{6} \pi_{6+1 / 2}(-0.3 / \varepsilon)$ \\
5 & $\sum_{n=0}^{5} a_{n} \varepsilon^{n}+(0.491496 \mathrm{E} 01) 6 !\left(\frac{\varepsilon}{0.05}\right)^{6} \Lambda_{6}(0.05 / \varepsilon)$ \\
10 & $\sum_{n=0}^{5} a_{n} \varepsilon^{n}+(0.977591 \mathrm{E} 01) 6 !\left(\frac{\varepsilon}{0.025}\right)^{6} \Lambda_{6}(0.025 / \varepsilon)$ \\
100 & $\sum_{n=0}^{5} a_{n} \varepsilon^{n}+(0.972859 \mathrm{E} 02) 6 !\left(\frac{\varepsilon}{0.0025}\right)^{6} \Lambda_{6}(0.0025 / \varepsilon)$
\end{tabular}

In this table the terminant $\Lambda$, also a tabulated function, is given by

$$
\Lambda_{m}(x)=\frac{1}{m !} \int_{0}^{\infty} \frac{\zeta^{m} \mathrm{e}^{-\zeta}}{1+\zeta / x} \mathrm{~d} \zeta .
$$

Dingle has also developed absolutely convergent expansions for the terminants $\Lambda$ and $\lambda$. Using them, absolutely convergent representations of the mean square response can be found and are given in Table 4.

In Table 4

$$
\begin{gathered}
\sum(\varepsilon)=\frac{-(0.3 / \varepsilon)}{6.5}\left[1+\frac{(0.3 / \varepsilon)}{5.5}+\frac{(0.3 / \varepsilon)^{2}}{(5.5)(4.5)}+\ldots\right] \\
\Omega(\varepsilon)=\frac{\pi(0.3 / \varepsilon)^{7.5} \exp (-0.3 / \varepsilon)}{(6.5) ! \tan 6.5 \pi} \\
\theta(f, \varepsilon)=6 !\left(\frac{\varepsilon}{f}\right)^{6}\left\{\frac{(f / \varepsilon)}{6}\left[1-\frac{(f / \varepsilon)}{5}+\ldots-\frac{(f / \varepsilon)^{5}}{5 !}\right]-\frac{(-f / \varepsilon)^{7}}{6 !} \sum_{t=0}^{\infty} \frac{\psi(t)-\ln (f / \varepsilon)}{t !}(f / \varepsilon)^{t}\right\} \\
\psi(t)=\frac{1}{\Gamma(t+1)} \frac{\mathrm{d}}{\mathrm{d} t} \Gamma(t+1)
\end{gathered}
$$

$\Gamma$ denoting the gamma function.

For purposes of comparison, calculations based on (i) linear system, (ii) first-order 
Table 4. Absolutely convergent expansion representation of the mean square response for various values of the intensity $D$

\begin{tabular}{cc}
\hline$D$ & $E\left[x^{2}\right]$ \\
\hline 0.0001 & $\sum_{n=0}^{5} a_{n} \varepsilon^{n}-(0.720118 \mathrm{E}-05)(6.5) !\left(\frac{\varepsilon}{0.3}\right)^{6}[\Sigma(\varepsilon)+\Omega(\varepsilon)]$ \\
0.001 & $\sum_{n=0}^{5} a_{n} \varepsilon^{n}-(0.719998 \mathrm{E}-04)(6.5) !\left(\frac{\varepsilon}{0.3}\right)^{6}[\Sigma(\varepsilon)+\Omega(\varepsilon)]$ \\
0.01 & $\sum_{n=0}^{5} a_{n} \varepsilon^{n}-(0.718805 \mathrm{E}-\Omega 3)(6.5) !\left(\frac{\varepsilon}{0.3}\right)^{6}[\Sigma(\varepsilon)+\Omega(\varepsilon)]$ \\
0.1 & $\sum_{n=0}^{5} a_{n} \varepsilon^{n}-(0.707305 \mathrm{E}-02)(6.5) !\left(\frac{\varepsilon}{0.3}\right)^{6}[\Sigma(\varepsilon)+\Omega(\varepsilon)]$ \\
5 & $\sum_{n=0}^{5} a_{n} \varepsilon^{n}+(0.491496 \mathrm{E} 01) \theta(0.05 \varepsilon)$ \\
10 & $\sum_{n=0}^{5} a_{n} \varepsilon^{n}+(0.977591 \mathrm{E} 01) \theta(0.025 \varepsilon)$ \\
100 & $\sum_{n=0}^{5} a_{n} \varepsilon^{n}+(0.972859 \mathrm{E} 02) \theta(0.0025 \varepsilon)$ \\
\hline
\end{tabular}

expansion, (iii) second-order expansion, and (iv) terminant expansion, were carried out for $\varepsilon=0.15$ and various values of $D$, and the results are shown in Table 5 .

Several observations can be made. Note that for values of $D \leqslant 0.1$ the linear portion of the system is dominant and that the first-order expansion essentially gives the response of the nonlinear system. The second-order and terminant expansions adjust the accuracy for the third and fourth significant figures, respectively. As the excitation increases in intensity,

Table 5. A comparison of mean square response $E\left[x^{2}\right]$ according to the linear system, first-order expansion, second-order expansion and terminant expansion for various values of intensity $D$

\begin{tabular}{|c|c|c|c|c|c|}
\hline \multirow[b]{2}{*}{$D$} & \multirow[b]{2}{*}{$\varepsilon$} & \multicolumn{4}{|c|}{$E\left[x^{2}\right]$} \\
\hline & & $\begin{array}{l}\text { Linear } \\
\text { system }\end{array}$ & $\begin{array}{l}\text { 1st order } \\
\text { expansion }\end{array}$ & $\begin{array}{l}\text { 2nd order } \\
\text { expansion }\end{array}$ & $\begin{array}{l}\text { Terminant } \\
\text { expansion }\end{array}$ \\
\hline 0.0001 & 0.015 & 0.1000000 E-03 & $0.9774932 \mathrm{E}-04$ & $0.9763965 \mathrm{E}-04$ & $0.9762209 \mathrm{E}-04$ \\
\hline 0.001 & 0.015 & $0.1000000 \mathrm{E}-02$ & $0.9774325 E-03$ & $0.9763372 E-03$ & $0.9761616 \mathrm{E}-03$ \\
\hline 0.01 & 0.015 & $0.1000000 \mathrm{E}-01$ & $0.9768250 \mathrm{E}-02$ & $0.9757441 \mathrm{E}-02$ & $0.9755693 \mathrm{E}-02$ \\
\hline 0.1 & 0.015 & $0.1000000 \mathrm{E} 00$ & $0.9707500 \mathrm{E}-01$ & $0.9698851 \mathrm{E}-01$ & $0.9697149 \mathrm{E}-01$ \\
\hline 5 & 0.015 & $0.5000000 \mathrm{E} 01$ & $0.3200000 \mathrm{E} 01$ & $0.4234438 E-01$ & $0.3756974 \mathrm{E} 01$ \\
\hline 10 & 0.015 & $0.1000000 \mathrm{E} 02$ & $0.3025000 \mathrm{E} 01$ & $0.1118153 \mathrm{E} 02$ & $0.3775957 \mathrm{E} 01$ \\
\hline 100 & 0.015 & $0.1000000 \mathrm{E} 03$ & $-0.5772500 \mathrm{E} 03$ & $0.7453455 \mathrm{E} 04$ & $-0.3772789 \mathrm{E} 07$ \\
\hline
\end{tabular}

significant deviations from the linear response occur, as can be seen from the results for $D \geqslant 5$. In these cases, decisions based on truncating the expansions at second order can be quite misleading, as the terminant expansion shows. With increasing values of $D$, the influence of the non-linear portion of the system can be expected to increase until eventually the basic underlying assumption that the solution can be represented as a perturbation about the linear solution is questionable. This is presumably what occurs when $D=100$, since the calculated negative value of the response is of course impossible, even though a linear pattern had been established on the inverted Domb-Sykes plot. To further pursue this point, the behavior of the system when $D=100$ was investigated for four other values of $\varepsilon$ and the results are shown in Table 6 . As expected, all responses are seen to be positive. For $\varepsilon=0.001$ and 0.005 note that the second-order expansions are inadequate and the terminant expansion is needed. Finally, observe that as $\varepsilon$ gets progressively smaller, the linear portion of the system again begins to dominate. 
Table 6. A comparison of mean square response $E\left[x^{2}\right]$ according to the linear system, first-order expansion, second-order expansion and terminant expansion for various values of $\varepsilon$

\begin{tabular}{lllccc}
\hline \multicolumn{1}{c}{} & \multicolumn{5}{c}{$E\left[x^{2}\right]$} \\
\hline$D$ & \multicolumn{1}{c}{$\varepsilon$} & $\begin{array}{c}\text { Linear } \\
\text { system }\end{array}$ & $\begin{array}{c}\text { 1st order } \\
\text { expansion }\end{array}$ & $\begin{array}{c}\text { 2nd order } \\
\text { expansion }\end{array}$ & $\begin{array}{c}\text { Terminant } \\
\text { expansion }\end{array}$ \\
\hline 100 & 0.001 & $0.1000000 \mathrm{E} \mathrm{03}$ & $0.5485000 \mathrm{E} \mathrm{02}$ & $0.9054202 \mathrm{E} \mathrm{02}$ & $0.6995111 \mathrm{E} \mathrm{02}$ \\
100 & 0.0005 & $0.1000000 \mathrm{E} \mathrm{03}$ & $0.7742500 \mathrm{E} \mathrm{02}$ & $0.8634801 \mathrm{E} \mathrm{02}$ & $0.8323705 \mathrm{E} \mathrm{02}$ \\
100 & 0.00025 & $0.1000000 \mathrm{E} \mathrm{03}$ & $0.8871250 \mathrm{E} \mathrm{02}$ & $0.9094325 \mathrm{E} \mathrm{02}$ & $0.9046707 \mathrm{E} \mathrm{02}$ \\
100 & 0.000125 & $0.1000000 \mathrm{E} \mathrm{03}$ & $0.9435625 \mathrm{E} \mathrm{02}$ & $0.9491394 \mathrm{E} \mathrm{02}$ & $0.9484574 \mathrm{E} \mathrm{02}$ \\
\hline
\end{tabular}

Acknowledgements-- The authors would like to thank the Rackham School of Graduate Studies of the University of Michigan for a Research Grant and to the Ford Motor Company for use of their computer facilities. Mr Michael Schoonmaker's aid in computing is also gratefully acknowledged.

\section{REFERENCES}

1. T. K. Caughey, Nonlinear theory of random vibrations, Adv. appl. Mech. 11, 209 (1971).

2. J. D. Atkinson, Eigenfunction expansions for randomly excited non-linear systems. J. Sound. Vib. 30,153 (1973).

3. H. J. Payne, The response of non-linear systems to stochastic excitation. Ph. D. Thesis, California Institute of Technology, (1967).

4. H. J. Payne, An approximate method for nearly linear, first order stochastic differential equations. Int. J. Control 7, 451 (1968).

5. M. Van Dyke, Analysis and improvement of perturbation series. Quart. J. appl. Mech. XXVII, 423 (1974).

6. R. B. Dingle, Asymptotic Expansions: Their Derivation and Interpretation. Academic Press, London (1973).

7. R. Courant and D. Hilbert, Methods of Mathematical Physics. Vol. I. Interscience (1955).

8. D. J. Buchanan, Analysis and improvement of divergent series. Quart. J. appl. Mech. XXIX, 127 (1976).

9. C. M. Bender and T. T. Wu, Anharmonic oscillator. Phys. Rev. 184, 1231 (1969).

Resume:

Dans ce travail, on s'interesse aux solutions en developpement de fonctions propres de l'equation directe de Fokker - Planck associee a un systeme particulier non lineaire du premier ordre soumis a une excitation avec un bruit blanc. En utilisant un ordinateur, on a genere un nombre substantiel de nouveaux termes dans les developpements. Avec cette nouvelle information, des graphiques inverses de Domb - Sykes revelent un modele dans les coefficients pour certains domaines de valeurs des parametres. On a alors utilise avec ce modele la theorie de Dingle pour refondre les series sous une forme plus favorable au calcul.

\section{Zusammenfassung:}

Diese Arbeit befasst sich mit Losungen mit Eigenfunktionsentwicklungen fur die vorwarts wirkende Gleichung nach Fokker und Planck, die ein bestimntes, nichtlineares System erster Ordnung unter Erregung durch weisses Rauschen, beschreibt. Mit Hilfe eines Digitalrechners wurde eine betrachtliche Anzahl neuer Gleider in der Entwicklung bestimnt. Mit dieser neuen Information zeigte sich in umgekehrten Domb-Sykes Diagrammen ein Muster in den Koeffizienten fur gewisse Zahlenbereiche der Parameter. Dingles Terminantentheorie wurde benutzt um mit Hilfe dieses Musters due Reihen in eine fur die Brechnung besser geeignete Form zu bringen. 